УДК 658.514

\author{
СИСТЕМА ПОЖАРОТУШЕНИЯ В НЕФТЯНОЙ \\ ПРОМЫШЛЕННОСТИ. МОДЕЛИ ТЕПЛОВОГО ИЗЛУЧЕНИЯ
}

\title{
FIRE EXTINGUISHING SYSTEM IN THE OIL INDUSTRY. THERMAL RADIATION MODELS
}

Ж.Н. Саласар, А.К. Чибас, А.В. Краснов, И.К. Бакиров

Уфимский государственный нефтяной технический университет, г. Уфа, Российская Федерация

Инжиниринговая компания нефтяных проектов, Гавана, Куба

Joel N. Salazar, Aryatna C. Chibas, Anton V. Krasnov, Irek K. Bakirov

Ufa State Petroleum Technological University, Ufa, Russian Federation

Engineering \& Oil Projects Company, Havana, Cuba

e-mail: salazarnoriega1987@gmail.com

Аннотация. Нефтяная промышленность, несомненно, является одной из наиболее уязвимых с точки зрения возникновения пожаров. В первую очередь, высокая пожарная опасность отрасли определяется большим количеством горючих веществ, которые обращаются в технологических процессах, поэтому системы пожаротушения имеют жизненно важное значение для этого типа промышленности.

Одним из наиболее часто встречающихся видов пожаров на объектах нефтяной промышленности являются возгорания в резервуарах для хранения топлива над поверхностью жидкости. Это явление известно на английском языке как Pool Fire (пожар зеркала резервуара).

При проектировании систем пожаротушения для резервуарных парков необходимо прорабатывать сценарии пожара, которые учитывают относительное пространственное положение каждого из резервуаров, 
чтобы в дальнейшем оценить систему охлаждения каждого из них (производить охлаждение резервуара по всему периметру или только по одной из его частей).

Главной целью анализа пространственного расположения резервуаров, а также расстояний между соседними резервуарами является определение зон защиты от теплового излучения, полученного при реализации пожара в одном из резервуаров рассматриваемого объекта. Сама защита обычно осуществляется путем устройства водяного орошения для резервуаров, расположенных в зоне риска, и включает частичное или полное охлаждение корпуса. Фактором, определяющим зону опасности воздействия теплового потока на объект, является интенсивность теплового излучения. В статье приведены значения интенсивности теплового излучения, которые являются критическими при воздействии на различные объекты.

Для оценки теплового излучения при горении легковоспламеняющихся жидкостей разработаны несколько математических моделей, дан анализ наиболее известных полуэмпирических моделей для оценки теплового излучения, возникающего при пожарах легковоспламеняющихся продуктов.

В результате сравнительного анализа предложено, в зависимости от расчетных значений интенсивности теплового излучения при пожаре, на нормативном уровне использовать модель точечного источника в качестве основного метода по оценке пространственного расположения резервуаров, а также расстояний между соседними резервуарами.

Abstract. The oil industry is undoubtedly one of the most dangerous for the occurrence of fires. First of all, the high fire hazard of the industry is determined by the large number of combustible substances that are circulated in technological processes, therefore fire extinguishing systems are vital for this type of industry. 
One of the most common types of fires at oil facilities is fire in storage tanks above the surface of the liquid. This phenomenon is known in English as Pool Fire (reservoir mirror fire).

When designing fire extinguishing systems for tank farms, it is necessary to work out fire scenarios that take into account the relative spatial position of each of the tanks in order to further evaluate the cooling system of each of them (to cool the tank around the entire perimeter or only one of its parts).

The main purpose of the analysis of the spatial location of the reservoirs, as well as the distances between adjacent reservoirs, is to determine the zones of protection against thermal radiation received during the implementation of a fire in one of the reservoirs of the object under consideration. The protection itself is usually carried out by means of a device for water irrigation for tanks located in the risk zone, and includes partial or complete cooling of the hull. The factor determining the zone of danger of exposure to heat flow to an object is the intensity of thermal radiation. The article presents the intensity thermal radiation, which are critical when exposed to various objects.

Several mathematical models have been developed for the assessment of thermal radiation during the combustion of flammable liquids, and an analysis of the most well-known semi-empirical models for the assessment of thermal radiation arising from fires of flammable products has been given.

As a result of a comparative analysis, it was proposed, depending on the calculated values of the thermal radiation intensity during a fire, to use the point source model at the normative level as the main method for estimating the spatial location of reservoirs, as well as the distances between adjacent reservoirs.

Ключевые слова: тепловое излучение, модель точечного источника, модель твердого пламени, критерий соседнего резервуара

Key words: thermal radiation, point source model, solid flame model, neighbor tank criterion 
Анализ пространственного расположения резервуаров, расстояний между соседними резервуарами необходим на ранних этапах проектирования для определения необходимых параметров системы пожаротушения. Главной целью анализа является определение зон защиты от теплового излучения, полученного при реализации пожара в одном из резервуаров рассматриваемого объекта. Сама защита обычно осуществляется путем устройства водяного орошения резервуаров, расположенных в зоне риска, и включает частичное или полное охлаждение корпуса.

Фактором, определяющим зону опасности воздействия теплового потока на объект, является интенсивность теплового излучения. Эта переменная выражается в единицах КВт/ ${ }^{2}$. Чтобы получить представление о значении интенсивности теплового излучения, можно сказать, что излучению в 1 КВт/м² человек подвергается в летний день. Длительное пребывание в подобных условиях может вызвать повреждения человеческого тела [1-5].

B IR-S-02 (1993) [6] определены значения интенсивности теплового излучения, которые являются критичсекими при воздействии на различные объекты (таблица 1).

В [6] также имеются данные для количественной оценки процентного повреждения оборудования, которое наступит при воздействии на него определенных значений интенсивности теплового излучения (таблица 2).

В [7] представлен критерий допустимого значения интенсивности теплового излучения (ASD). Определены два критерия: один для зданий и сооружений - значение интенсивности излучения составляет 31,5 кВТ/ м², и другой для людей - значение интенсивности излучения составляет $1,4 \kappa \mathrm{BT} / \mathrm{M}^{2}$. 
Таблица 1. Значения интенсивности теплового излучения и возможные повреждения [6]

\begin{tabular}{|c|l|}
\hline $\begin{array}{c}\text { Интенсивность теплового излучения } \\
\left(\text { кВт/м }{ }^{2}\right)\end{array}$ & \multicolumn{1}{|c|}{ Виды повреждений } \\
\hline 12,00 & Плавление пластика \\
\hline 13,50 & $\begin{array}{l}\text { Минимальная энергия для повреждения } \\
\text { материалов с низкой температурой плавления. } \\
\text { Это значение используется для разделения } \\
\text { конических баков крыши }\end{array}$ \\
\hline 18,00 & Деградация пластика \\
\hline 22,10 & $\begin{array}{l}\text { Безопасный предел для хранения сжиженного } \\
\text { газа }\end{array}$ \\
\hline 37,50 & Повреждение технологического оборудования \\
\hline
\end{tabular}

Таблица 2. Вероятность повреждения оборудования

\begin{tabular}{|c|c|}
\hline $\begin{array}{c}\text { Процент повреждения оборудования } \\
(\%)\end{array}$ & Интенсивность теплового излучения (кВт/м²) \\
\hline 1 & 12.10 \\
\hline 50 & 21.50 \\
\hline 99 & 38.00 \\
\hline Библиографическая информация: IR-S-02 (1993)[6] \\
\hline
\end{tabular}

Пожар зеркала резервуара возникает в результате того, что источник тепла вводится в верхнюю свободную область резервуара, где скапливаются испарившиеся пары пожароопасных жидкостей и также присутствует кислород, который является окислителем химической реакции горения. Данная свободная зона на поверхности зеркала жидкости в резервуаре может рассматриваться пространственно как круглая зона, так как она отражает форму вертикальных стальных резервуаров [5].

Для оценки теплового излучения при горении легковоспламеняющихся жидкостей разработаны несколько математических моделей. Наиболее известные представлены в таблице 3.

Из приведенных в таблице 3 моделей чаще всех применяются модель точечного источника (MFP) и модель твердого пламени (MLLS). Распространенность применения модели точечного источника (MFP) объясняется ее простотой и доступностью применения, а модель твердого 
пламени получила наибольшее распространения в связи с тем, что лучше описывает реальность пожара и переноса теплового потока [5].

Таблица 3. Полуэмпирические модели для оценки теплового излучения, возникающего при пожарах легковоспламеняющихся продуктов

\begin{tabular}{|c|c|}
\hline \multirow[b]{2}{*}{ Пунктуальные модели } & модель точечного источника (MFP) \\
\hline & $\begin{array}{l}\text { модель многочисленных источников } \\
\text { (МFРМ) }\end{array}$ \\
\hline \multirow{3}{*}{$\begin{array}{l}\text { Модели } \\
\text { на диффузионной } \\
\text { поверхности }\end{array}$} & модель «твердого» пламени (MLLS) \\
\hline & $\begin{array}{l}\text { модель эквивалентного источника } \\
\text { теплового потока (MRE) }\end{array}$ \\
\hline & $\begin{array}{l}\text { модель приблизительного эквивалентного } \\
\text { источника теплового потока (MREA) }\end{array}$ \\
\hline
\end{tabular}

Величина излучаемого теплового потока зависит от множества факторов, которые могут быть сгруппированы следующим образом:

1. свойства топлива (энтальпия горения и испарения, температура кипения и удельная теплоемкость жидкости);

2. характеристики пламени (температура, удельная эмиссионная мощность, геометрия и высота пламени и диаметр разлива);

3. другие химические процессы, связанные с горением: образование паро- и газообразных продуктов, частично сжигаемых, которые уменьшают излучающую способность пламени.

Природа вещества и геометрические характеристики пламени играют решающую роль в определении значений интенсивности теплового излучения. Во-первых, потому что пламя определяет поток пара, поступающего в огонь, хотя пар также зависит от механизмов испарения, которые происходят во время события, а, во-вторых, потому что он характеризует размер источника излучения [8].

С другой стороны, тепловое излучение, производимое горящим объектом, также зависит от характеристик приемника (типа, размеров, местоположения и ориентации относительно пламени) и погодных условий (влажность, состав воздуха (углекислый газ уменышают передачу 
теплового потока)), ветер охлаждает объект и меняет направление передачи тепла [5].

Модель точечного источника (MFP) характеризуется группой гипотез:

1. процесс горения сосредоточен в одной точке, из которой испускается вся излучаемая энергия;

2. для каждого вещества излучаемая энергия представляет собой долю от общей энергии, образующейся в процессе горения;

3. распространение теплового потока описывает сферическую геометрию, которая имеет в качестве источника точку, в которой концентрируется пламя.

C учетом вышеуказанных ограничений интенсивность теплового излучения, воздействующего на объект, расположенный на расстоянии х от центра резервуара, может быть рассчитана следующим образом:

$$
I_{x}=\frac{\tau_{a} * F_{S} * S * m * \Delta H_{c}}{4 * \pi * x^{2}}
$$

где $I_{X}-$ интенсивность теплового излучения, воздействующего на объект, расположенный на расстоянии х от центра резервуара, кВт/м²;

$\tau_{a}-$ коэффициент пропускаемости атмосферы. Безразмерный параметр, значение колеблется от 0 до 1 ;

$F_{S}-$ фракция излучаемой энергии. Безразмерный параметр, значение колеблется от 0,1 до 0,4 ;

m' - массовый расход испарений на единицу площади, кг/ ${ }^{2} \cdot c$;

$\mathrm{S}$ - площадь горения, $\mathrm{M}^{2}$;

$\Delta H_{C}-$ энтальпия реакции горения, кДж/кг;

X - расстояние от центра пламени до облучаемого объекта, м.

В уравнении (1) коэффициент пропускания атмосферы зависит от х и нескольких атмосферных факторов в соответствии с TNO (2005) [9]:

$$
\tau_{a}=1-\alpha_{W}-\alpha_{C}
$$


где $\alpha_{W}$ и $\alpha_{C}$ - аддитивные коэффициенты поглощения водяного пара и двуокиси углерода из атмосферы, поскольку они являются основными компонентами воздуха, поглощающего тепловое излучение.

Известно, что $\alpha_{C}$ очень мал по сравнению с $\alpha_{W}$, поэтому практика использования показывает, что его приравнивают к нулю [5].

Данные по коэффициенту $\alpha_{W}$ приведены графически в [5]. Однако, чтобы получить необходимую информацию из графика, в начале нужно определить соотношение между $\mathrm{P}_{\mathrm{W}}$ и $\mathrm{x}\left(\mathrm{P}_{\mathrm{W}}\right.$ определяется ниже). Данный источник также указывает на то, что расчетная температура горения принимается равной значению $1200 \mathrm{~K}$ (усредненная температура горения углеводородов). Следуя этой логике, мы получили уравнение:

$$
\alpha_{W}=a+b \sqrt{\left(P_{W} * x\right)}+c\left(P_{W} * x\right)^{1.5}+e\left(P_{W} * x\right)^{2}+f\left(P_{W} * x\right)^{2.5}+g\left(P_{W} * x\right)^{3},
$$

где $a=0,02301848$;

$$
\begin{aligned}
& b=0,0018476 ; \\
& c=5,3036 * 10^{-6} ; \\
& e=1,062 * 10^{-11}, \\
& f=6,41567 * 10^{-15}, \\
& g=1,6239 * 10^{-18},
\end{aligned}
$$

Для расчета частичного давления водяного пара в воздухе $P_{W}$ представлены уравнения (4) и (5). Эти уравнения можно найти в [9] и [8] соответственно:

$$
\begin{gathered}
P_{W}=P_{W O} * \frac{H_{r}}{100}, \\
P_{W}=1013.25 * H_{r} * e^{\left(14.4114-\frac{5328}{T_{a}}\right)},
\end{gathered}
$$

где $P_{W}$ - частичное давление водяного пара в воздухе, Па;

$P_{W o}$ - давление пара, насыщенное водой в воздухе, Па;

$H_{r}$ - относительная влажность, \%;

$T_{a}$ - температура окружающей среды, К. 
Модель твердого пламени (MLLS) была первоначально предложена Raj (1977), но впервые опубликована только в [8] при расчете пожара зеркала резервуара. Хотя данную модель рекомендовано использовать при расчетах Pool Fire, еe можно применять при оценке любого типа пожара, и в настоящее время она является одной из самых строгих методологий для определения интенсивности теплового излучения.

Согласно [8], модель твердого пламени (MLLS) базируется на следующих основных принципах:

1. Пламя представляет собой простую трехмерную геометрию, такую как цилиндры, листы, сферы, конусы и т.д., размеры которых соответствуют размерам пожара;

2. лучистая энергии теплового потока распространяется равномерно по наружной поверхности пламени, принимая во внимание как эмитенту только видимой области.

С учетом этих соображений мы имеем, что в модели твердого пламени интенсивность падающего излучения в объекте, расположенном на расстоянии х от центра резервуара, вычисляется по формуле:

$$
I_{X=} \tau_{a} * F_{\text {máx }} * S E P_{a c t},
$$

где $F_{m a ́ x}-$ это так называемый максимальный коэффициент зрения. Безразмерный параметр;

$S E P_{a c t}$ - текущая эмиссионная мощность поверхности, кВт/м².

Максимальный коэффициент видимости $F_{m a ́ x}$ можно определить с помощью следующего уравнения:

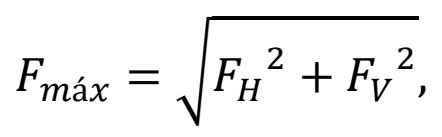

где $F_{H}$ - визуальный компонент на горизонтальной оси;

$F_{V}$ - визуальный компонент на вертикальной оси. 
Коэффициент видимости $F_{m a ́ x}$ оценивается обычно в сложных выражениях, которые зависят от геометрии пламени, расстояния до экспонированного объекта и относительной ориентации [10].

Твердая модель пламени обычно устанавливается как круговой базовый цилиндр, а ее высота равна высоте пламени.

В этой модели определяются два случая, первый так называемый MLLS без присутствия ветра и второй MLLS с присутствием ветра (рисунок 1, $a$ и б соответственно).

В обоих случаях $R$ и $D$ являются соответственно радиусом и диаметром сгоревшего резервуара, х является расстоянием от центра резервуара до облучаемого объекта. В случае отсутствия ветра средняя высота пламени $L$ направлена по вертикали (рисунок 1, a), при наличии ветра высота пламени следует за своей конфигурацией, то есть в направлении, которое фиксирует угол $\theta$, измеренный от вертикального направления. Наконец, $u_{w}$ является скоростью ветра (рисунок 1, б).

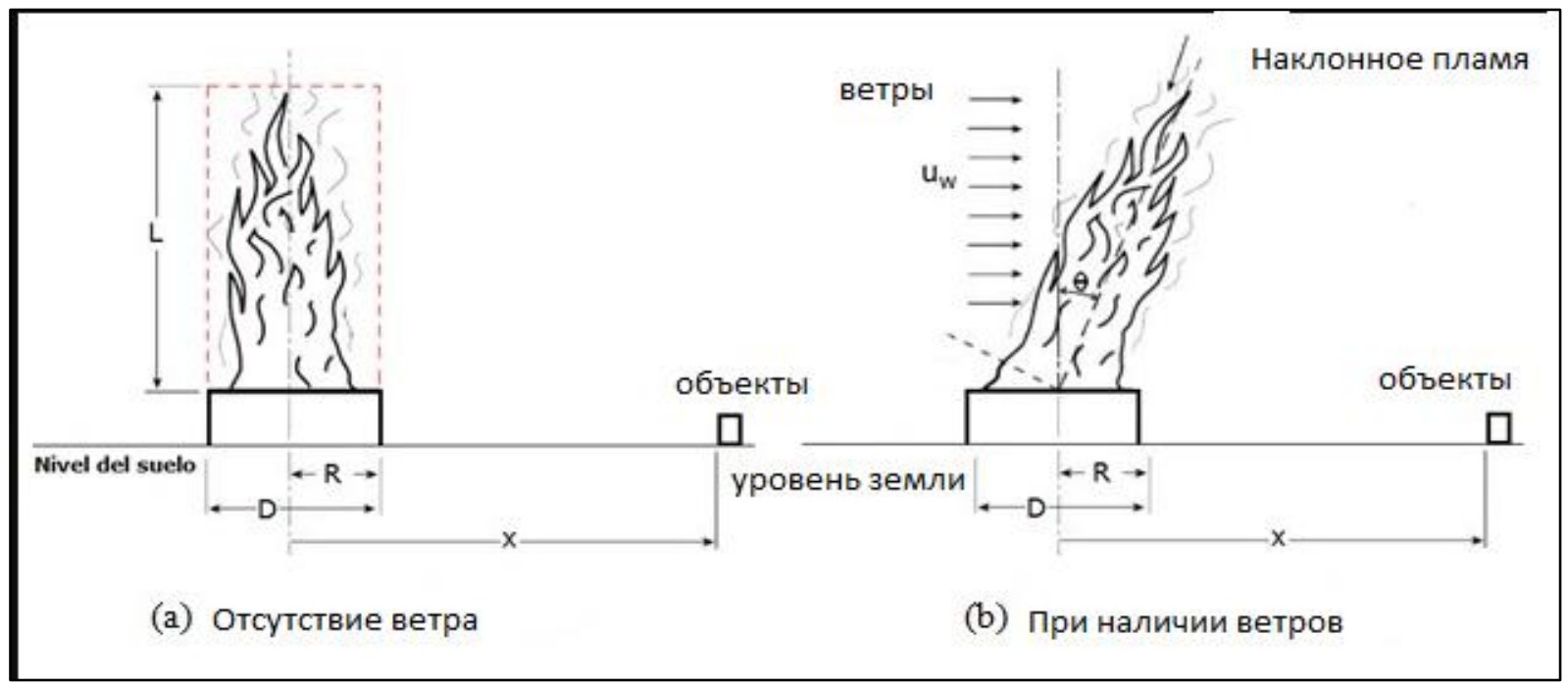

Рисунок 1. Профили пожаров в резервуаре для хранения топлива при наличии ветра и без него

Модель MLLS без наличия ветра (TNO (2005)) представлена на рисунке 2. 


$$
\begin{gathered}
x_{r}=\frac{x}{R} \\
h_{r}=\frac{L}{R} \\
A=\left(x_{r}+1\right)^{2}+h_{r}^{2} \\
B=\left(x_{r}-1\right)^{2}+h_{r}^{2} \\
F_{h}=\frac{1}{\pi}\left\{\left[\tan ^{-1}\left(\sqrt{\frac{x_{r}+1}{x_{r}-1}}\right)\right]-\left[( \frac { x _ { r } ^ { 2 } - 1 + h _ { r } ^ { 2 } } { \sqrt { A \cdot B } } ) \cdot \operatorname { t a n } ^ { - 1 } \left(\sqrt{\left.\left.\left.\frac{\left(x_{r}-1\right) A}{\left(x_{r}+1\right) B}\right)\right]\right\}}\right.\right.\right. \\
\frac{1}{\pi \cdot x_{r}}\left\{\left[\tan ^{-1}\left(\frac{h_{r}}{\sqrt{x_{r}^{2}-1}}\right)\right]-\left[h_{r} \cdot \tan ^{-1}\left(\sqrt{\frac{x_{r}-1}{x_{r}+1}}\right)\right]+\ldots\right. \\
\ldots\left[( \frac { h _ { r } ( A - 2 \cdot x _ { r } ) } { \sqrt { A \cdot B } } ) \cdot \operatorname { t a n } ^ { - 1 } \left(\sqrt{\left.\left.\left.\frac{\left(x_{r}-1\right) A}{\left(x_{r}+1\right) B}\right)\right]\right\}}\right.\right.
\end{gathered}
$$

Рисунок 2. Уравнения, используемые в модели твердого пламени без ветра

Для расчета средней высоты пламени $L$ было предложено много моделей. Как правило, для видимого пламени в условиях спокойствия можно использовать уравнение Томаса 1963 г., цитируемое TNO (2005) [9], в котором отношение $L / D$ является функцией $m^{\prime}, \rho_{a}, D$ :

$$
L=42 *\left(\frac{m}{\rho_{a} * \sqrt{g * D}}\right)^{0.61} * D
$$

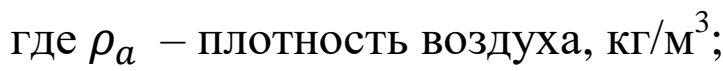

$g$ - ускорение силы тяжести, м/ $\mathrm{c}^{2}$;

$D$ - диаметр танкера, м.

Модель MLLS с учетом направления и силы ветра представлена на рисунке 3 (TNO (2005)).

Уравнения для определения интенсивности падающего излучения на объекте в модели твердого пламени с ветром (TNO (2005) [9]) представлены на рисунке 4. 
Чтобы вычислить среднюю высоту пламени $(L)$ при оценках с учетом ветра, уравнение Томаса дополняется параметром термина $u^{*}$, который известен как безразмерная скорость ветра.

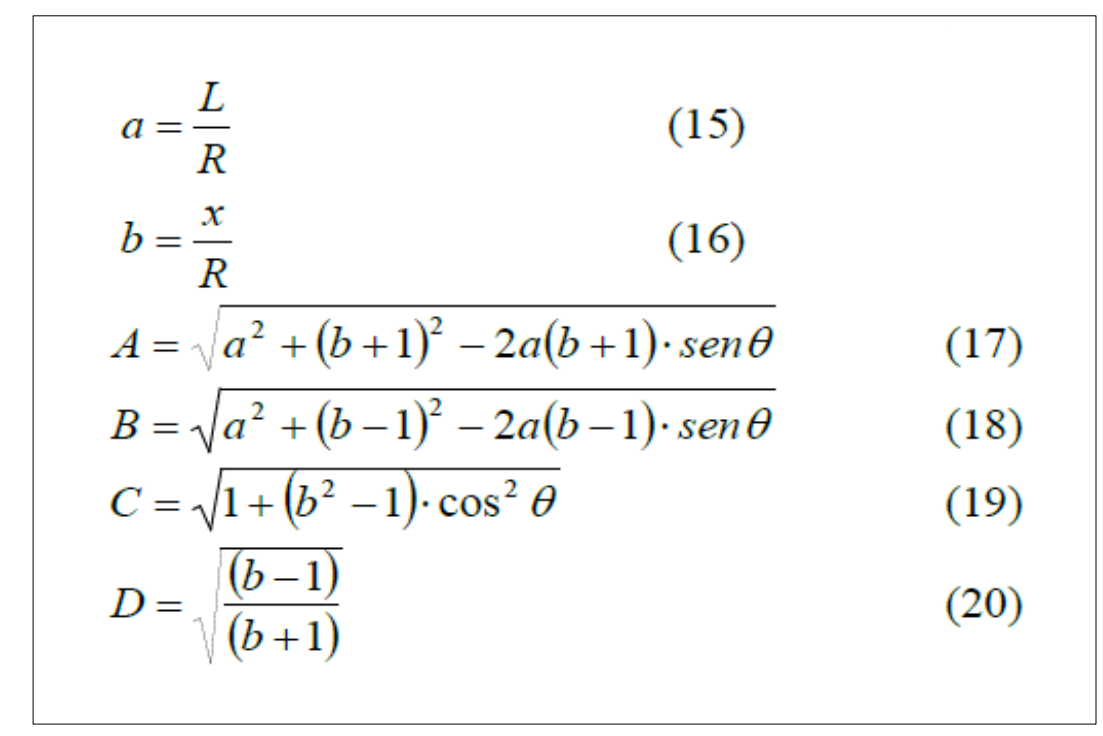

Рисунок 3. Уравнения, используемые в модели твердого пламени с ветром согласно TNO (2005)

$\begin{aligned} & E=\frac{a \cdot \cos \theta}{b-a \cdot \operatorname{sen} \theta} \text { F= } \sqrt{\left(b^{2}-1\right)} \\ & F_{h}= \frac{1}{\pi}\left\{\tan ^{-1}\left(\frac{1}{D}\right)+\frac{\operatorname{sen} \theta}{C}\left[\tan ^{-1}\left(\frac{(a \cdot b)-F^{2} \cdot \operatorname{sen} \theta}{F \cdot C}\right)+\tan ^{-1}\left(\frac{F^{2} \cdot \operatorname{sen} \theta}{F \cdot C}\right)\right]-\ldots\right. \\ & {\left.\left[\left(\frac{a^{2}+(b+1)^{2}-2(b+1+a \cdot b \cdot \operatorname{sen} \theta)}{A \cdot B}\right) \cdot \tan ^{-1}\left(\frac{A \cdot D}{B}\right)\right]\right\} } \\ & F_{v}=\frac{1}{\pi}\left\{\left[-E \cdot \tan ^{-1}(D)\right]+\left[E \cdot\left(\frac{a^{2}+(b+1)^{2}-2 b \cdot(1+a \cdot \operatorname{sen} \theta)}{A \cdot B}\right) \cdot \tan ^{-1}\left(\frac{A \cdot D}{B}\right)\right]+\ldots\right. \\ & \ldots {\left.\left[\frac{\cos \theta}{C} \cdot\left[\tan ^{-1}\left(\frac{a \cdot b-F^{2} \cdot \operatorname{sen} \theta}{F \cdot C}\right)+\tan ^{-1}\left(\frac{F^{2} \cdot \operatorname{sen} \theta}{F \cdot C}\right)\right]\right]\right\} }\end{aligned}$

Рисунок 4. Уравнения, предложенные TNO (2005) [9] для определения интенсивности падающего излучения на объекте в модели твердого пламени с ветром 
В этом случае уравнение выглядит следующим образом:

$$
L=55 *\left(\frac{m}{\rho_{a} * \sqrt{g * D}}\right)^{0.67} *\left(u^{*}\right)^{-0.21} * D
$$

Для получения значения $u^{*}$ необходимо определить характерную скорость ветра uс:

$$
u c=\left[9.81 * m *\left(\frac{D}{\rho_{a}}\right)\right]^{\frac{1}{3}}
$$

Если $u_{w}<u_{c}$, то $u^{*}=1$,

Если $u_{w}>u_{c}$, то $u^{*}=u_{w} / u_{c}$,

Для скорости ветра $u_{w}>u_{c}$ высота пламени имеет тенденцию оставаться постоянной.

Для расчета угла наклона пламени относительно вертикали $\theta$ можно сначала использовать последовательность, рекомендованную Американской ассоциацией газа (1974), цитируемую [9], в которой если $u^{*} \geq 1$, то угол получается путем применения уравнения:

$$
\theta=\cos ^{-1} \times\left(\frac{1}{\sqrt{u^{*}}}\right)
$$

Если $u^{*}<1$, то принимается $\cos \theta=1$, затем $\theta=0$ (условия спокойствия) [5].

Если $u^{*}<1$ и в этих условиях $\cos \theta=1$, то $\theta=0$ (условия спокойствия) [5].

В [9] также предлагается уравнение:

$$
\theta=\operatorname{sen}^{-1} \times\left[\frac{\sqrt{\left(4 \times c^{2}\right)+1}-1}{2 \times c}\right],
$$

где параметр $c$ может быть вычислен с помощью уравнения (29), в котором коэффициенты $a^{\prime}, b^{\prime}, C^{\prime}$ и $d^{\prime}$ заданы для разных условий в таблице 4:

$$
c=a^{\prime} \times\left(N_{F}\right)^{b^{\prime}} \times\left(N_{R}\right)^{C^{\prime}} \times\left(\frac{\rho_{V}}{\rho_{a}}\right)^{d^{\prime}},
$$




$$
\begin{aligned}
& N_{F}=\frac{u_{W}^{2}}{(g \times D)}, \\
& N_{R}=\frac{D \times u_{W}}{v_{a}},
\end{aligned}
$$

где $N_{F}-$ число Фруда, безразмерный параметр;

$N_{R}$ - число Рейнольдса, безразмерный параметр;

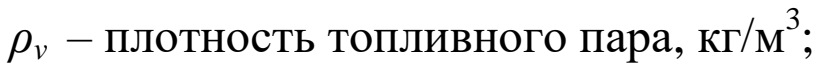

$v_{a}-$ кинематическая вязкость воздуха, $\mathrm{m}^{2} / \mathrm{c}$.

Поверхностная эмиссионная мощность рассчитывается в соответствии со следующими уравнениями:

$$
\begin{gathered}
S E P_{a c t}=\left[S E P_{\text {máx }} \times(1-\zeta)\right]+\left[S E P_{\text {soot }} \times \zeta\right], \\
S E P_{\text {máx }}=\frac{F_{S} \times m^{\prime} \times \Delta H_{c}}{\left[1+\left(4 \times \frac{L}{D}\right)\right]}
\end{gathered}
$$

где $\mathrm{SEP}_{\text {máx }}$ - максимальная мощность излучения, кВт / м² ;

$\mathrm{SEP}_{\text {soot }}-$ объемная эмиссия углеродистой сажи, кВт / $\mathrm{M}^{2}$ (согласно [9] еe значение может быть установлено на уровне 20 кВт / ${ }^{2}$ );

ろ - для лужевых пожаров 0,8 согласно [9].

Таблица 4. Параметры уравнения (29) [10]

\begin{tabular}{|l|c|c|c|c|}
\hline & $a^{\prime}$ & $b^{\prime}$ & $c^{\prime}$ & $d^{\prime}$ \\
\hline Коническое пламя & 3,00 & 0,422 & 0,011 & 0 \\
\hline Цилиндрическое пламя & 1,90 & 0,399 & 0,050 & 0 \\
\hline Sliepcevich (1996) & 3,30 & 0,80 & 0,07 & 0 \\
\hline Binding (1992) & 0,666 & 0,333 & 0,117 & $-0,60$ \\
\hline
\end{tabular}

\section{Применение моделей}

Данные по топливам:

Бензин

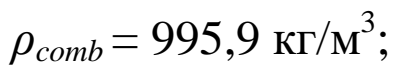

$\Delta H c=39800$ кДж/кг;

$m^{\prime}=0,035 \kappa г / \mathrm{M}^{2} \mathrm{c} ;$

Данные по резервуарам: 
$D=58$ м (диаметр), $H=18,75$ м (высота), $V o l=50000$ м $^{3}$ (объем).

Aтмосферные данные (только для MLLS с присутствием ветра):

$H_{r}=80 \%$;

$T_{t}=28{ }^{\circ} \mathrm{C}$;

$\rho_{\text {aire }}=1,1732 \mathrm{~kg} / \mathrm{m}^{3}$;

$v_{\text {aire }}=1,327 * 10-5 \mathrm{~m}^{2} / \mathrm{s}$;

$P_{w o}=3994,29 \mathrm{~Pa}$;

$u_{w}=5 \mathrm{~m} / \mathrm{s}$;

Другие:

$F s=0,4$

$S E P_{\text {soot }}=20 \mathrm{\kappa BT} / \mathrm{M}^{2}$;

$\zeta=0.8$.

\section{Результатьл}

В случае использования модели точечного источника (MPF) частичное давление водяного пара в воздухе рассчитывается с помощью уравнения (4), $P_{w}=3195,43 P_{a}$.

При использовании модели твердого пламени (MLLS) без присутствия ветра средняя высота пламени рассчитывается по уравнению (14), $L=41,30 \mathrm{м}$, а также рассчитывается $S E P_{\text {máx }}=144,80 \mathrm{\kappa BT} / \mathrm{M}^{2}$ и $S E P_{a c t}=44,96 \mathrm{\kappa BT} / \mathrm{M}^{2}$.

В расчете по модели MLLS с наличием ветра получены следующие значения $u_{c}=2,57 \mathrm{~m} / \mathrm{c}, u^{*}=1,945 \mathrm{~m} / \mathrm{c}, L=31,49$ м, угол наклона пламени рассчитывается по выражениям (27) и (28) $\theta=44,20^{\circ}$ и $\theta=48,43^{\circ}$ соответственно, для выражения (28) было получено, что $N_{F}=0,439$ и $N_{R}=21851066$, кроме того, вычисляются $S E P_{\text {máx }}=175,68 \mathrm{\kappa BT} / \mathrm{M}^{2}$ и $S E P_{a c t}=51,15 \kappa \mathrm{BT} / \mathrm{M}^{2}$.

Другие параметры зависят от расстояния (от Х до М), в связи с этим было сделано изменение этой переменной от стенки резервуара примерно до 190 м. Расчеты произведены для трех рассмотренных моделей, результаты можно оценить по рисунку 5. 
Как видно, все графики начинаются на расстоянии 29 м от геометрического центра резервуара или на границе стенки резервуара. На этом расстоянии модели MLLS с учетом ветром и без учета имеют значения интенсивности теплового излучения, равные 35,40 и 22,39 кВт/м² соответственно, однако расчеты по модели MFP показывают значительное увеличение интенсивности - 101,55 кВт/м². По мере увеличения расстояния между горящим и соседним резервуарами все модели, как правило, сходятся, поскольку влияние геометрии пламени снижается.

Исходя из данных таблицы 1 для определения безопасного расстояния между резервуарами, принимаем в качестве «безопасного» значения интенсивности теплового излучения величину 10 кВт/м². На графике 2 (рисунок 5) можно заметить, что это значение $I=10 \mathrm{\kappa BT} / \mathrm{m}^{2}$ достигается на расстоянии 21 м, 39 м и 61,50 м, измеренных от стенки горящего резервуара, согласно моделям MLLS без ветра, MLLS с ветром и MFP соответственно.

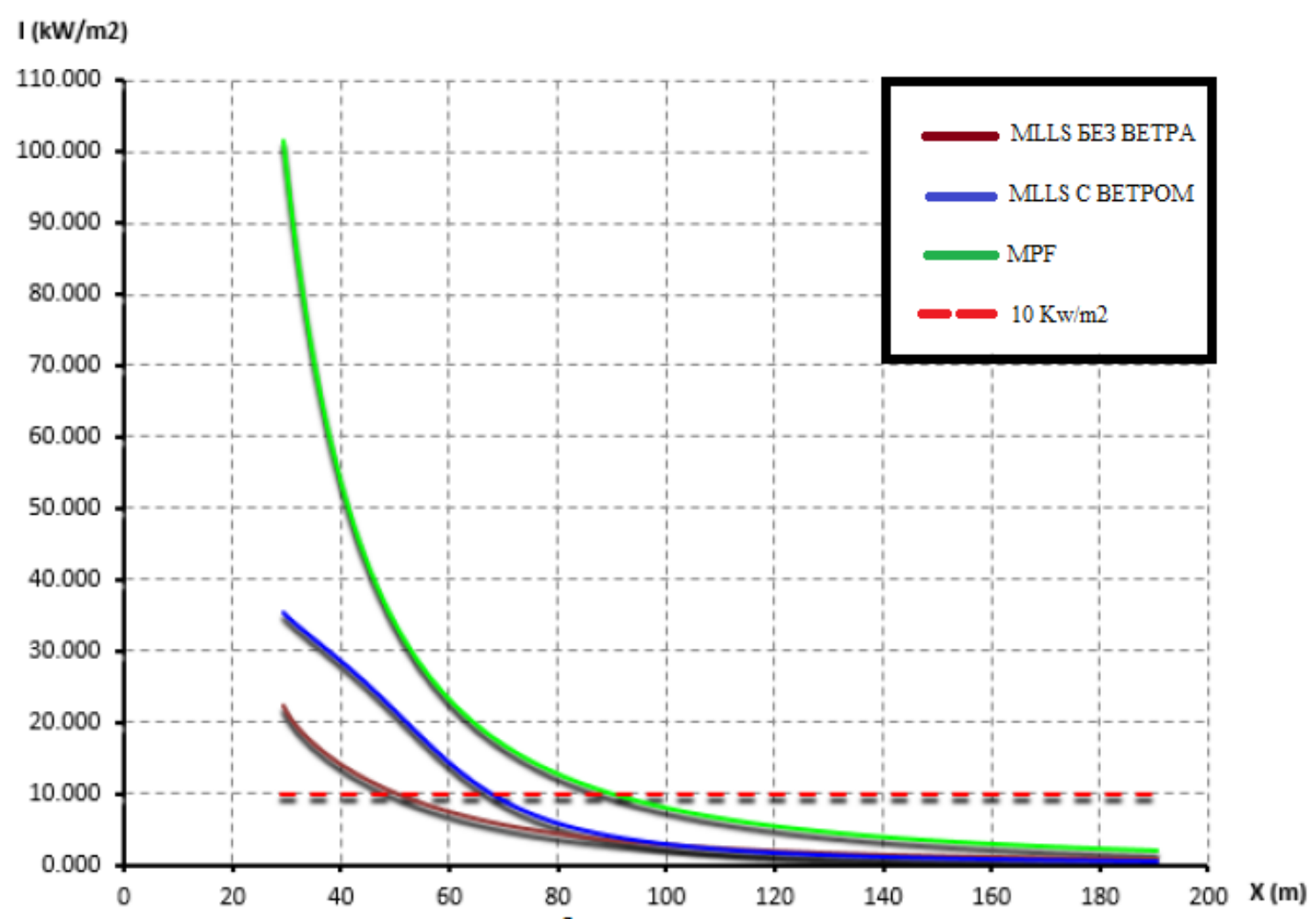

Рисунок 5. График зависимости $I$ (кВт / м $\left.^{2}\right)$ от X (м) для радиационных моделей 


\section{Выводы}

1. Значение $I=10 \mathrm{\kappa BT} / \mathrm{M}^{2}$ принимается в качестве предельной интенсивности теплового излучения при оценке безопасного расстояния между резервуарами для хранения топлива.

2. Модель точечного источника (MFP) имеет ряд преимуществ в сравнении с моделями MLLS. Во-первых, она проще в реализации, так как не учитывает геометрические параметры пламени. Во-вторых, модель точечного источника имеет более высокие расчетные значения, что позволяет обеспечить задел безопасности при нормировании расстояний между резервуарами хранения нефти.

3. На нормативном уровне предлагается использовать модель точечного источника (MFP) в качестве основного метода оценки пространственного расположения резервуаров, а также расстояний между соседними резервуарами, в зависимости от расчетных значений интенсивности теплового излучения при пожаре.

\section{Список используемых источников}

1. Alomá B.A. Integration of Models for the Calculation of Thermal Radiation and Flow under pressure in Piping Networks for the Design of Fire Extinguishing Systems in the Oil Industry / Undergraduate, Faculty of Civil Engineering. José Antonio Echeverría Higher Polytechnic Institute (CUJAE), Havana, Cuba, 2013.

2. Bustamante G.F. Calculations of the Heat Flux of Radiation (Version 1.03). Technical Reference Guide. APCI Fire Protection Agency, Havana, Cuba, 2012.

3. IR-M-03. Fire Extinguishing System, PDVSA-Petróleos de Venezuela, SA. Caracas, Venezuela, 1983. 
4. NC 96-02-18. Base Storage of Oil and its Derivatives. The System of Standards of Fire Protection. State Committee for Standardization. Central Level. Cuba, 1987.

5. González F.E., Ruiz F.J., Miñana A.A., Navarro G.J., Ruiz G.Jy, Martínez A.J. Planning Areas for Severe Thermal Accidents (under Royal Decree 1254/99 (Seveso II) / Department of Chemical Engineering. University of Murcia. General Directorate of Civil Protection, Ministry of the Interior. Spain, 2002.

6. IR-S-02. Criteria for Quantitative Risk Analysis, PDVSA-Petróleos de Venezuela, SA. Caracas, Venezuela, 1993.

7. McGrattan B.K., Baum R.H., Hamins A. Thermal Radiation from Large Pool Fires. National Institute of Standards and Technology. U.S. Department of Commerce. USA, 2000.

8. CPQRA. Quantitative Analysis of the Risks of Chemical Processes / Chemical Process Safety Center of the American Institute of Chemical Engineers. Second Edition. 3 Park Avenue, New York, USA, 2000.

9. TNO. Methods for Calculating Physical Impacts due to Emissions of Hazardous Materials (Liquids and Gases) // Yellow Book CPR-14E. Netherlands Organization for Applied Scientific Research. Holland, 2005.

10. Fleury R. Evaluation of Thermal Radiation Models for the Propagation of Fire between Objects: Master Thesis in Fire Engineering / Faculty of Civil and Natural Resources, University of Canterbury. Christchurch, New Zealand, 2010.

\section{References}

1. Alomá B.A. Integration of Models for the Calculation of Thermal Radiation and Flow under pressure in Piping Networks for the Design of Fire Extinguishing Systems in the Oil Industry. Undergraduate, Faculty of Civil Engineering. José Antonio Echeverría Higher Polytechnic Institute (CUJAE), Havana, Cuba, 2013. 
2. Bustamante G.F. Calculations of the Heat Flux of Radiation (Version 1.03). Technical Reference Guide. APCI Fire Protection Agency, Havana, Cuba. 2012.

3. IR-M-03. Fire Extinguishing System, PDVSA-Petróleos de Venezuela, SA. Caracas, Venezuela. 1983.

4. NC 96-02-18. Base Storage of Oil and its Derivatives. The System of Standards of Fire Protection. State Committee for Standardization. Central Level. Cuba, 1987.

5. González F.E., Ruiz F.J., Miñana A.A., Navarro G.J., Ruiz G.Jy, Martínez A.J. Planning Areas for Severe Thermal Accidents (under Royal Decree 1254/99 (Seveso II). Department of Chemical Engineering. University of Murcia. General Directorate of Civil Protection, Ministry of the Interior. Spain, 2002.

6. IR-S-02. Criteria for Quantitative Risk Analysis. PDVSA-Petróleos de Venezuela, SA. Caracas, Venezuela, 1993.

7. McGrattan B.K., Baum R.H., Hamins A. Thermal Radiation from Large Pool Fires. National Institute of Standards and Technology. U.S. Department of Commerce. USA, 2000.

8. CPQRA. Quantitative Analysis of the Risks of Chemical Processes. Chemical Process Safety Center of the American Institute of Chemical Engineers. Second Edition. 3 Park Avenue, New York, USA, 2000.

9. TNO. Methods for Calculating Physical Impacts due to Emissions of Hazardous Materials (Liquids and Gases). Yellow Book CPR-14E. Netherlands Organization for Applied Scientific Research. Holland, 2005.

10. Fleury R. Evaluation of Thermal Radiation Models for the Propagation of Fire between Objects: Master Thesis in Fire Engineering / Faculty of Civil and Natural Resources, University of Canterbury. Christchurch, New Zealand, 2010. 


\section{Сведения об авторах}

\section{About the authors}

Жоэль Норьега Саласар, студент кафедры «Пожарная и промышленная безопасность», УГНТУ, г. Уфа, Российская Федерация

Joel N. Salazar, Student of Fire and Industrial Safety Department, USPTU, Ufa, Russian Federation

e-mail: salazarnoriega1987@gmail.com

Ариатна Кастаньера Чибас, мастер по добыче нефти и газа Инжиниринговой Компании нефтяных проектов, г. Гавана, Куба

Aryatna Castaner Chibas, Master in Oil and Gas Production of the Engineering \& Oil Projects Company, Havana, Cuba

Краснов Антон Валерьевич, канд. техн. наук, доцент кафедры «Пожарная и промышленная безопасность», УГНТУ, г. Уфа, Российская Федерация

Anton V. Krasnov, Candidate of Engineering Sciences, Assistant Professor of Fire and Industrial Safety Department, USPTU, Ufa, Russian Federation e-mail:00770088@mail.ru

Бакиров Ирек Климович, канд. техн. наук, доцент, доцент кафедры «Пожарная и промышленная безопасность», УГНТУ, г. Уфа, Российская Федерация

Irek K. Bakirov, Candidate of Engineering Sciences, Associate Professor, Assistant Professor of Fire and Industrial Safety Department, USPTU, Ufa, Russian Federation

e-mail: bakirovirek@bk.ru 